\title{
Best One-Sided Multiplier Approximation of Unbounded Functions by Trigonometric Polynomials
}

\author{
Saheb K. A-Saidy, Naseif J. Al-Jawari and Raad F. Hassan* \\ Department of Mathematics, College of Science, University of Al-Mustansiryh, Baghdad-Iraq
}

Article's Information

Received:

27-10-2021

Accepted:

16-11-2021

Published:

31-12-2021

\section{Keywords:}

Multiplier convergence

Multiplier integral

Multiplier modulus one-sided multiplier

DOI: 10.22401/ANJS.24.4.06

*Corresponding author: raadfhassanabod@gmail.com

\section{Introduction}

Babenko in 2012) [1] estimated the rate of best one-sided approximation of characteristic polynomials. In 2015, Soltani F. [2] studied some classes of Dunkl multiplier operators and gave an application of the theory reproducing kernels to Tikhonov of regularization which gives the best approximation of the operators on Hilbert space. In 2017 , Saheb Kehaid Jassim and Abeer M. Salih [3] studied the multiplier approximation of unbounded functions by Bernstein-Durrmeyer operators.

In 2020, Saheb Al-Saidy and Ali H. Zaboon [4], obtained the multiplier approximation of periodical unbounded functions by Trigonometric operators. In 2020, A. M. Pasko [5], studied the point wise estimation of onesided approximation of the class $W_{\infty}^{r}, 0<r<1$.

\section{Basic Concepts}

Definition 1, [6]. A series $\sum_{n=0}^{\infty} a_{n}$ is called a multiplier convergent if there is a sequence $\left\{\psi_{n}\right\}_{n=0}^{\infty}$, such that $\sum_{n=0}^{\infty} a_{n} \psi_{n}<\infty$ and we will say that $\left\{\psi_{n}\right\}_{n=0}^{\infty}$ is a multiplier for the convergence.

Definition 2, [4]. For any real valued function $f$ defined on $X=[0,2 \pi]$ if here is a sequence $\left\{\psi_{n}\right\}_{n=0}^{\infty}$, such that $\int_{X} f \psi_{n}(x) \mathrm{d} x<\infty, X=[0,2 \pi]$, then we say that $\psi_{n}$ is the multiplier integral.

Definition 3. Let $f \in L p, \psi_{n}(X), X=[0,2 \pi], p \in[1, \infty)$ be the space of all real valued unbounded functions $f$, such that $\int_{X} f \psi_{\mathrm{n}}(x) \mathrm{d} x<\infty$, then the norm:

\section{Abstract}

$\|f\|_{L p, \psi_{n}(X)}=\left(\int_{X}\left|f \psi_{n}(x)\right|^{p}\right)^{1 / p}, x \in X$ is the norm on $L p, \psi_{n}(X)$

For $f \in L p, \psi_{n}(X), X=[0,2 \pi], 0<\delta$, we will define the following concepts:

$\omega(f, \delta) p, \psi_{n}=\sup _{|h|<\delta}\|f(x+h)-f(x)\|_{L p, \psi_{n}(X)}$ the multiplier modulus of continuity of function $f$

$\tau_{k}(f, \delta) p, \psi_{n}=\left\|\omega_{k}(f, \delta)\right\|_{L p, \psi_{n}(X)}, p \in[1, \infty), k \in \mathbb{N}$ is the multiplier averaged modulus of smoothness of $f$ of order $k$, where the $k^{\text {th }}$ modulus of smoothness.

The smoothness for $f$ is defined by:

$$
\begin{gathered}
\omega(f, x, \delta) p, \psi_{n}=\sup _{|h|<\delta}\left\{\left\{\left\|\Delta_{h}^{k} f(f, \mathrm{t})\right\|_{L p, \psi_{n}(X)}: t,\right.\right. \\
\left.t+k h \in\left[x-\frac{k \delta}{2}, x+\frac{k \delta}{2}\right]\right\}
\end{gathered}
$$

where The $k^{\text {th }}$ symmetric difference of function $f$ is:

$$
\Delta_{h}^{k} f(x)=\sum_{i=0}^{k}\left(\begin{array}{l}
k \\
i
\end{array}\right)(-1)^{k-i} f\left(x-\frac{k \delta}{2}+i h\right), x \mp \frac{k h}{2} \in X
$$

Definition 4, [7]. For $f \in L p(X), X=[\mathrm{a}, \mathrm{b}], 0<\delta$, we will define the following concepts:

$$
\omega(f, \delta) p=\sup _{|h|<\delta}\|f(x+h)-f(x)\|_{L p(X)}
$$

the modulus of continuity of function $f$

$$
\tau_{k}(f, \delta) p=\left\|\omega_{k}(f, \delta)\right\|_{L p(X)}, p \in[1, \infty), k \in \mathbb{N}
$$

is the averaged modulus of smoothness of $f$ of order $k$. where the $k^{\text {th }}$ modulus of smoothness.

The smoothness for $f$ is defined by:

$\omega(f, x, \delta) p=\sup _{|h|<\delta}\left\{\Delta_{h}^{k} f(f, \mathrm{t}) \|_{L p(X)}: t, t+k h \in\right.$ $\left.\left[x-\frac{k \delta}{2}, x+\frac{k \delta}{2}\right]\right\}$

where the $k^{\text {th }}$ symmetric difference of function $f$ is:

$$
\Delta_{h}^{k} f(x)=\sum_{i=0}^{k}\left(\begin{array}{l}
k \\
i
\end{array}\right)(-1)^{k-i} f\left(x-\frac{k \delta}{2}+i h\right), x \mp \frac{k h}{2} \in X
$$




\section{Al-Nahrain Journal of Science}

ANJS, Vol.24 (4), December, 2021, pp. 40-45

Definition 5. For $f \in L p, \psi_{n}(X), X=[0,2 \pi], 0<\delta$, we will define the following concepts:

$\omega(f, \delta) p, \psi_{n}=\sup _{|h|<\delta}\|f(x+h)-f(x)\|_{L p, \psi_{n}(X)}$ the multiplier modulus of continuity of function $f$

$\tau_{k}(f, \delta) p, \psi_{n}=\left\|\omega_{k}(f, \delta)\right\|_{L p, \psi_{n}(X)}, p \in[1, \infty), k \in \mathbb{N}$ is the multiplier averaged modulus of smoothness of $f$ of order $k$, where the $k^{\text {th }}$ modulus of smoothness.

The smoothness for $f$ is defined by: $\omega(f, x, \delta) p, \psi_{n}=\sup _{|h|<\delta}\left\{\left\|\Delta_{h}^{k} f(f, \mathrm{t})\right\|_{L p, \psi_{n}(X)}: \mathrm{t}, \mathrm{t}+k\right.$ $\left.h \in\left[x-\frac{k \delta}{2}, x+\frac{k \delta}{2}\right]\right\}$

Where The $k^{\text {th }}$ symmetric difference of function $f$ is $\Delta_{h}^{k} f(x)=\sum_{i=0}^{k}\left(\begin{array}{l}k \\ i\end{array}\right)(-1)^{k-i} f\left(x-\frac{k \delta}{2}+i h\right), x \mp \frac{k h}{2} \in X$

Definition 6, [8]. The degree of best one - sided approximation of $f$ is:

$\tilde{E}_{n}(f) p=\inf \left\{\left\|\mathrm{p}_{n}-\mathrm{q}_{n}\right\|_{L p(X)}: q_{n}(x) \leq f(x) \leq p_{n}(x)\right\}$

Also, the degree of best approximation of a function $f \in$ $L p(X)$ is define by:

$$
E_{n}(f) p=\inf \left\{\left\|f-\mathrm{p}_{n}\right\|_{L p(X)}: p_{n} \in P_{n}\right\}
$$

Definition 7. The degree of best one-sided multiplier approximation of $f$ is:

$\tilde{E}_{n}(f) p, \psi_{n}=\inf \left\{\left\|\mathrm{p}_{n}-\mathrm{q}_{n}\right\|_{L p, \psi_{n}(X)}: q_{n}(x) \leq f(x) \leq\right.$ $\left.p_{n}(x)\right\}$

Also, the degree of best multiplier approximation of a function $f \in L p, \psi_{n}(X)$ is define by:

$E_{n}(f) p, \psi_{n}=\inf \left\{\left\|f-\mathrm{p}_{n}\right\|_{L p, \psi_{n}(X)}: p_{n} \in P_{n}\right\}$

\section{Auxiliary Lemmas}

Here we will study some propositions of a functions in the space $L p, \psi_{n}(X), X=[0,2 \pi]$.

Lemma 1. Let $f \in L p, \psi_{n}(X), X=[0,2 \pi]$, then:

$E_{n}(f) p, \psi_{n} \leq \widetilde{E}_{n}(f) p, \psi_{n}$

Proof. Consider $p_{n}^{*}$ to be the best polynomial approximation of $f$ and let $q_{n}, p_{n}$ be the best one-sided poly of $f$, where:

$$
\begin{aligned}
q_{n}(x) \leq f(x) & \leq p_{n}(x) \\
E_{n}(f) p, \psi_{n} & =\inf \left\{\left\|f-p_{n}\right\|_{L p, \psi_{n}(X)}: p_{n} \in \mathrm{P}_{n}\right\} \\
& =\left\|f-\mathrm{p}_{n}^{*}\right\|_{L p, \psi_{n}(X)} \\
& =\left(\int_{\mathrm{x}}\left|\left(f-p_{n}^{*}\right)(x) \psi_{n}(x)\right|^{p} \mathrm{~d} x\right)^{1 / p} \\
& \leq\left(\int_{\mathrm{X}}\left|\left(\mathrm{p}_{n}-q_{n}\right)(x) \psi_{n}(x)\right|^{p} \mathrm{~d} x\right)^{1 / p} \\
& =\left\|p_{n}-q_{n}\right\|_{p, \psi_{n}} \\
& =\widetilde{E}_{n}(f) p, \psi_{n} .
\end{aligned}
$$

lemma 2. Let $f \in L p, \psi_{n}(X), X=[0,2 \pi]$, then:

$$
\tilde{E}_{n}(f)_{p, \psi_{n}} \leq \mathrm{c} p E_{n}(f)_{p, \psi_{n}}
$$

Proof. Consider $p^{*} \in \mathrm{P}$ is the best approximation of $f \in$ $L p, \psi_{n}(X)$ and let $s_{1}, s_{2} \in P_{n}$ be the best one-sided approximation of $f$ such that $s_{2}(x) \leq f(x) \leq s_{1}(x)$

$$
f(x) \leq s_{1}(x)
$$

$$
\begin{aligned}
& s_{1}(x) \leq 2 f(x), s_{m}(x), s_{n}(x) \in P_{n} \\
& \tilde{E}_{n}(f)_{p, \psi_{n}}=\inf \left\|s_{n}-s_{m}\right\|_{L p, \psi_{n}(X)} \\
& =\left\|s_{1}-s_{2}\right\|_{L p, \psi_{n}(X)} \\
& =\left(\int_{\mathrm{x}}\left|\left(s_{1}-s_{2}\right)(x) \psi_{n}(x)\right|^{p} d x\right)^{1 / p} \\
& =\left(\int_{\mathrm{X}}\left|\left[s_{1}(x)-s_{2}(x)\right] \psi_{n}(x)\right|^{p} d x\right)^{1 / p} \\
& \leq\left(\int_{\mathrm{X}}\left|[2 f(x)-f(x)] \psi_{n}(x)\right|^{p} d x\right)^{1 / p} \\
& =\left(\int_{\mathrm{X}} \mid\left[2 f(x)-p^{*}(x)+p^{*}(x)-\right.\right. \\
& \left.f(x)]\left.\psi_{n}(x)\right|^{p} d x\right)^{1 / p} \\
& =\left(\int_{\mathrm{x}}\left|\left[2 f(x)-p^{*}(x)\right] \psi_{n}(x)\right|^{p} d x\right)^{1 / p}+ \\
& \left(\int_{\mathrm{x}}\left|\left[f(x)-p^{*}(x)\right] \psi_{n}(x)\right|^{p} d x\right)^{1 / p} \\
& =\left\|2 f-p^{*}\right\|_{L p, \psi_{n}(X)}+\left\|f-p^{*}\right\|_{L p, \psi_{n}(X)} \\
& \leq 2 E_{n}(f)_{p, \psi_{n}} \text {. }
\end{aligned}
$$

Lemma 3. Let $f, \mathrm{~g}, \varphi \in L p, \psi_{n}(X), X=[0,2 \pi]$ be $2 \pi$ periodic functions, if:

$$
\left|[f(x)-g(x)] \psi_{n}(x)\right| \leq \varphi(x) \psi_{n}(x)
$$

Then:

$$
\begin{gathered}
\tilde{E}_{n}(f) p, \psi_{n} \leq C p\left(\tilde{E}_{n}(\mathrm{~g}) p, \psi_{n}+2 \tilde{E}_{n}(\varphi) p, \psi_{n}+\right. \\
\left.2\|\varphi\|_{L p, \psi_{n}(X)}\right)
\end{gathered}
$$

where $C$ is constant depends on $p$.

Proof. Let $p_{n}^{*}$ is the best approximation of $f$ and $g_{n}^{*}$ is the best approximation of $\varphi$

$$
\begin{aligned}
& E_{n}(f) p, \psi_{n}= \inf \left\|f-p_{n}\right\|_{L p, \psi_{n}(X)}, p_{n} \in \mathrm{T}_{\mathrm{n}} \\
&=\left\|f-p_{n}^{*}\right\|_{L p, \psi_{n}(X)} \\
&\left\|f-p_{n}^{*}\right\|_{L p, \psi_{n}(X)}=\left(\int_{\mathrm{x}}\left|\left(f-p_{n}^{*}\right) \psi_{n}(x)\right|^{p} d x\right) \\
&=\left(\int_{\mathrm{X}} \mid\left(f+g+\varphi+g_{n}-g-\varphi-\right.\right. \\
&\left.\left.\quad g_{n} p_{n}^{x}\right)\left.\psi_{n}(x)\right|^{p} d x\right)^{1 / p} \\
& \leq\left(\int_{\mathrm{X}}\left|\left(g-p_{n}^{*}\right) \psi_{n}(x)\right|^{p} d x\right)^{1 / p}+ \\
&\left(\int_{\mathrm{X}} \mathrm{I}\left|(f-g) \psi_{n}(x)\right|^{p} d x\right)^{1 / p}+ \\
&\left(\left.\int_{\mathrm{X}} \mathrm{I}\left(\varphi-g_{n}^{*}\right) \psi_{n}(x)\right|^{p} d x\right)^{1 / p}+ \\
&\left(\left.\int_{\mathrm{X}} \mathrm{I}\left(\varphi-g_{n}^{*}\right) \psi_{n}(x)\right|^{p} d x\right)^{1 / p} \\
& \leq\left\|\left(g-p_{n}^{*}\right)\right\|_{L p, \psi_{n}(X)}+\|(f- \\
&g)\left\|_{L p, \psi_{n}(X)}+\right\|\left(\varphi-g_{n}^{*}\right) \|_{L p, \psi_{n}(X)}+ \\
&\left\|\left(\varphi+g_{n}^{*}\right)\right\|_{L p, \psi_{n}(X)} \\
& \leq E_{n}(g)_{p, \psi_{n}}+E_{n}(f) p, \psi_{n}+\|(\varphi)\| p, \psi_{n}+ \\
& E_{n}(\varphi) p, \psi_{n}+E_{n}(\varphi) p, \psi_{n}
\end{aligned}
$$

from Lemma 1 and Lemma 2, we get:

$$
\begin{aligned}
& E_{n}(f) p, \psi_{n} \leq \tilde{E}_{n}(g) p, \psi_{n}+2\|\varphi\|_{L p, \psi_{n}(X)}+ \\
& 2 \widetilde{E}_{n}(\varphi)_{p, \psi_{n}} \\
& \tilde{E}_{n}(f) \leq C\left(\tilde{E}_{n}(g)_{p, \psi_{n}}+2\|(\varphi)\| p, \psi_{n}+2 \tilde{E}_{n}(\varphi)_{p, \psi_{n}}\right)
\end{aligned}
$$

Lemma 4. Let $f \in L p, \psi_{n}(X), X=[0,2 \pi]$, then: $\omega(f, \delta) p, \psi_{n} \leq \delta\|f\|^{\prime} p, \psi_{n}$

Proof.

$$
\begin{aligned}
\omega(f, \delta) p, \psi_{n} & =\sup \left\{\left|[f(x+h)-f(x)] \psi_{n}(t) \quad\right|:\right. \\
& |h| \leq \delta, x, x+h \in X\} \\
= & \left|\int_{x}^{x+h} f(\mathrm{t}) \psi_{n}(\mathrm{t}) d t\right|
\end{aligned}
$$




\section{Al-Nahrain Journal of Science}

ANJS, Vol.24 (4), December, 2021, pp. 40-45

$$
\begin{aligned}
& \leq \sup \int_{x}^{x+h}\left|f^{\prime}(t) \psi_{n}(x)\right| \mathrm{d} t \\
& \leq|h| \sup ^{\prime}\left|f^{\prime}(x) \psi_{n}(x)\right| \\
& \leq|h|\left\|f^{\prime}\right\|_{p, \psi_{n}} \\
& \leq \delta\left\|f^{\prime}\right\|_{p, \psi_{n}} .
\end{aligned}
$$

Lemma 5. Let $f \in L p, \psi_{n}(X), X=[0,2 \pi], 1 \leq \mathrm{p}<\infty$, then:

$E_{n}(f) p, \psi_{n} \leq \mathrm{C}(\kappa) \omega_{k}(f, \delta) p, \psi_{n}, \delta>0$

Proof. Let $p_{n}^{*}$ is the best approximation of $f$, where $\mathrm{T}_{\mathrm{n}}$ is the set of all trigonometric polynomial.

$E_{n}(f) p, \psi_{n}=\inf \left\|f-p_{n}\right\|_{L p, \psi_{n}(X)}, p_{n} \in \mathrm{T}_{\mathrm{n}}$

$$
\begin{aligned}
= & \left\|f-p_{n}^{*}\right\|_{L p, \psi_{n}(X)} \\
& =\left(\int_{\mathrm{x}}\left|\left(f-p_{n}^{*}\right)(x) \psi_{n}(x)\right|^{p} d x\right)^{1 / p}
\end{aligned}
$$

$$
\leq \sup \left(\int_{\mathrm{X}} \mathrm{I}\left[\left.\left(f(x)-p_{n}^{*}(x)\right] \psi_{n}(x)\right|^{p} d x\right)^{1 / p}\right.
$$$$
=\sup \left(\int_{\mathrm{X}} \mid\left[\left.(f(x)-f(x+h)] \psi_{n}(x)\right|^{p} d x\right)^{1 / p}\right.
$$$$
=c_{p} \sup \left(\int_{\mathrm{x}}\left|\Delta_{h}^{k} f(x) \psi_{n}(x)\right|^{p} d x\right)^{1 / p}
$$$$
=c_{p} \sup \left\|\Delta_{h}^{k} f(x)\right\|_{L p, \psi_{n}(X)} \text {. }
$$$$
=c_{p} \omega_{k}(f, \delta) p, \psi_{n}
$$

Then:

$$
E_{n}(f) p, \psi_{n} \leq c_{p} \omega_{k}(f, \delta) p, \psi_{n} .
$$

Lemma 6. Let $f \in L p, \psi_{n}(X)$, then:

$\omega_{k}(f, \delta) p, \psi_{n} \leq \omega_{k-1}(f, \delta) p, \psi_{n}$ if $f$ exists.

Proof.

$$
\begin{aligned}
\Delta_{h}^{k} f(x) \psi_{n}(x) & =\Delta_{h}^{k-1} \Delta_{h} f(x) \psi_{n}(x) \\
& =\Delta_{h}^{k-1}\left[(f(x+\mathrm{h})-f(x)) \psi_{n}(x)\right] \\
& =\Delta_{h}^{k-1} \int_{0}^{h}\left(f(x+\mathrm{t}) \psi_{n}(x) \mathrm{dt}\right.
\end{aligned}
$$

$\left|\Delta_{h}^{k-1} f(x) \psi_{n}(x)\right|=\mid\left(f^{\prime}(x+\mathrm{t}) \psi_{n}(x) \mathrm{d} t \mid\right.$

$$
\begin{aligned}
& \leq \int_{\min (0, h)}^{\max (0, h)}\left|\Delta_{h}^{k-1} f^{\prime}(x+\mathrm{t}) \psi_{n}(x)\right| \mathrm{d} t \\
& \leq \int_{\min (o, h)}^{\max (o, h)} \omega_{k-1}\left(f^{\prime}, \delta\right) p, \psi_{n} \mathrm{~d} t \\
& \leq h \omega_{k-1}(\dot{f}, \delta) p, \psi_{n} \\
& \leq \delta \omega_{k-1}\left(\dot{f}^{\prime}, \delta\right) p, \psi_{n}
\end{aligned}
$$

$\omega_{k}(f, \delta) p, \psi_{n}=\delta \omega_{k-1}(f, \delta) p, \psi_{n}$

Lemma 7. Let $f \in L p, \psi_{n}(X)$, then: $\omega_{k}(f, \delta) p, \psi_{n} \leq \delta^{k}\left\|f^{(k)}\right\| p, \psi_{n}$

Proof. From Lemma 6 and Lemma 4

$$
\begin{aligned}
\omega_{k}(f, \delta) p, \psi_{n} & \leq \delta \omega_{k-1}\left(f^{\prime}, \delta\right) p, \psi_{n} \\
& \leq \delta \delta \omega_{k-2}(f, \delta) p, \psi_{n} \\
& \leq \delta^{k-1} \omega\left(f^{(k-1}, \delta\right) p, \psi_{n} \\
& \leq \delta^{k-1} \delta\left\|f^{(k)}\right\| p, \psi_{n} \\
& \leq \delta^{k}\left\|f^{(k)}\right\| p, \psi_{n}
\end{aligned}
$$

Lemma 8. Let $f \in L p, \psi_{n}(X), f^{(k)} \in L p, \psi_{n}(X)$

Then:

$$
\widetilde{E}_{n}(f) p, \psi_{n} \leq c_{k} n^{-k}\left\|f^{(k)}\right\| p, \psi_{n}, n>k
$$

Proof. From Lemmas 2, 5 and Lemma 7, we get:

$\tilde{E}_{n}(f) p, \psi_{n} \leq C p E_{n}(f) p, \psi_{n}$

$$
E_{n}(f) p, \psi_{n} \leq C(k) \omega_{k}(f, \delta) p, \psi_{n}
$$

$\omega_{k}(f, \delta) p, \psi_{n} \leq \delta^{k}\left\|f^{(k)}\right\| p, \psi_{n}$

\section{Then:}

$\tilde{E}_{n}(f) p, \psi_{n} \leq C(k) \delta^{k}\left\|f^{(k)}\right\| p, \psi_{n}$

Lemma 9. Let $f \in L p, \psi_{n}(X), X=[0,2 \pi]$, with $\omega_{k}(f, x, h) p, \psi_{n}$ is a function of $x$, then:

$$
\tau_{1}\left(\omega_{\mathrm{k}}(f, x, h), \delta\right) p, \psi_{n} \leq \tau_{\mathrm{k}}\left(f, h+\frac{\delta}{k}\right) p, \psi_{n}
$$

Proof. Set $g(x)=\omega_{k}(f, x, h) p, \psi_{n}$

$\omega_{1}(\mathrm{~g}, x, \delta) p, \psi_{n}=\sup \left\{\left|\Delta_{\theta} g(t) \psi_{n}(t)\right|: t, t+\theta \in[x-\right.$

$$
\begin{aligned}
&\left.\frac{\delta}{2}, x+\frac{\delta}{2}\right] \\
&= \sup \left\{\left|[g(t+\theta)-g(t)]_{\psi n}\right|: t, t+\theta \in[x-\right. \\
&\left.\left.\frac{\delta}{2}, x+\frac{\delta}{2}\right]\right\} \\
& \leq \sup \left\{g(t): t \in\left[x-\frac{\delta}{2}, x+\frac{\delta}{2}\right]\right\} \\
&= \sup \left\{\operatorname { s u p } \left\{\left|\Delta_{m}^{k} f(s) \psi_{n}(s)\right|: \mathrm{s}, \mathrm{s}+k m \in\left[t-\frac{\delta}{2},\right.\right.\right. \\
&\left.\left.\left.t+\frac{\delta}{2}\right]\right\}: t \in\left[x-\frac{\delta}{2}, x+\frac{\delta}{2}\right]\right\} \\
& \leq \sup \left\{\left|\Delta_{m}^{k} f(s) \psi_{n}(s)\right|: s, s+k m \in\left[x-\frac{\delta}{2}\right.\right. \\
&\left.\left.-\frac{k h}{2}, x+\frac{\delta}{2}-\frac{k h}{2}\right]\right\} \\
&= \omega_{k}\left(f, x, h+\frac{k}{2}\right) p, \psi_{n}
\end{aligned}
$$

$\|\omega(g, x, s)\| p, \psi_{n} \leq\left\|\omega_{k}\left(f, x, h+\frac{\delta}{k}\right)\right\| p, \psi_{n}$

$\tau_{1}(\mathrm{~g}, x, h, \delta) p, \psi_{n} \leq \tau_{\mathrm{k}}\left(f, x h+\frac{\delta}{k}\right) p, \psi_{n}$

$\tau_{1}\left(\omega_{k}(f, x, h), \delta\right) p, \psi_{n} \leq \tau_{\mathrm{k}}\left(f, x, h+\frac{\delta}{k}\right) p, \psi_{n}$

Lemma 10. Let $L p, \psi_{n}(X), X=[0,2 \pi], g_{n}(x)=$ $\omega_{k}\left(f, x, n^{-1}\right) p, \psi_{n}$, then:

$\widetilde{E}_{n}\left(g_{n}\right) p, \psi_{n} \leq C \tau_{\mathrm{k}}\left(f, 2 n^{-1}\right) p, \psi_{n}$

where $C$ is a constant.

Proof. From Lemma 9, letting $h=\delta=\mathrm{n}^{-1}$, we have:

$$
\tau_{1}\left(g_{n}, n^{-1}\right) p, \psi_{n} \leq \tau_{\mathrm{k}}\left(f, n^{-1}\right) p, \psi_{n}
$$

from Theorem 1

$$
\begin{aligned}
E_{n}^{\sim}\left(g_{n}\right) p, \psi_{n} & \leq C \tau_{1}\left(g_{n}, n^{-1}\right) p, \psi_{n} \\
& \leq C \tau_{k}\left(f, 2 n^{-1}\right) p, \psi_{n}
\end{aligned}
$$

Lemma 11. Let $f \in L p, \psi_{n}(X), f^{\prime}$ exists, then:

$\tau_{\mathrm{k}}(f, \delta) p, \psi_{n} \leq \tau_{k-1}\left(f^{\prime}, \frac{\delta}{k-1} \delta\right) p, \psi_{n}$

Proof. Since:

$$
\begin{aligned}
& \Delta_{h}^{k}\left[f(t) \psi_{n}(t)\right]=\Delta_{h}^{k-1} \Delta_{h}\left[f(t) \psi_{n}(t)\right] \\
& =\Delta_{h}^{k-1}\left([f(\mathrm{t}+h)-f(\mathrm{t})] \psi_{n}(t)\right) \\
& =\Delta_{h}^{k-1}\left(\int_{0}^{h}\left[\hat{f}(u+t) \psi_{n}(u)\right] d u\right), h>0
\end{aligned}
$$

$\left|\Delta_{h}^{k}\left[f(t) \psi_{n}(t)\right]\right| \leq \int_{0}^{h}\left|\Delta_{h}^{k-1} \hat{f}(u+t) \psi_{n}(u)\right| \mathrm{d} u$ $\sup \left\{\left|\Delta_{h}^{k}\left[f(t) \psi_{n}(t)\right]\right|: \mathrm{t}, \mathrm{t}+k h \in\left[x-\frac{k \delta}{2}, x+\frac{k \delta}{2}\right]\right.$

$$
\leq \sup \left\{\int_{0}^{h}\left|\Delta_{h}^{k-1} \hat{f}(u+t) \psi_{n}(u) d u\right|\right\} \cap[\mathrm{a}, \mathrm{b}]
$$

$\omega_{k}(f, x ; \delta) p, \psi_{n} \leq h \omega_{k^{-1}}\left(\dot{f}^{\prime}, x ; \delta\right) p, \psi_{n}$

$$
\leq \delta \omega_{\mathrm{k}-1}(\dot{f}, x ; \delta) p, \psi_{n}
$$

$\left\|\omega_{k-1}(f, x ; \delta)\right\| p, \psi_{n} \leq \delta\left\|\omega_{k-1}(f, x ; \delta)\right\| p, \psi_{n}$ $\tau_{\mathrm{k}}(f, \delta) p, \psi_{n} \leq \delta \tau_{k-1}\left(\dot{f}, \frac{k}{k-1} \delta\right) p, \psi_{n}$

Lemma 12. Let $f \in L p, \psi_{n}(X), X=[0,2 \pi]$

$\tau_{1}(f, \delta) p, \psi_{n} \leq \delta\left\|f^{\prime}\right\| p, \psi_{n}, \delta \in[0,2 \pi]$ 


\section{Al-Nahrain Journal of Science}

ANJS, Vol.24 (4), December, 2021, pp. 40-45

\section{Proof.}

$\omega(f, x, \delta) p, \psi_{n}=\sup \left\{\left|[f(t)-f(\hat{t})] \psi_{n}(t)\right|: \mathfrak{t}, \hat{\mathrm{t}} \in\right.$

$$
\begin{aligned}
& {\left.\left[x-\frac{\delta}{2}, x+\frac{\delta}{2}\right]\right\} } \\
= & \sup \left\{\left|\int_{t}^{t} f(t) \psi_{n}(t) d t\right|: \mathfrak{t}^{\prime}, \dot{\mathrm{t}} \in\left[x-\frac{\delta}{2}, x+\frac{\delta}{2}\right]\right\} \\
\leq & \int_{x-\frac{\delta}{2}}^{x+\frac{\delta}{2}}\left|\hat{f}(t) \psi_{n}(t)\right| \mathrm{d} t \\
= & \leq \int_{-\frac{\delta}{2}}^{\frac{\delta}{2}}\left|\hat{f}(x+t) \psi_{n}(t)\right| \mathrm{d} t
\end{aligned}
$$

$\|\omega(f, x ; \delta)\| p, \psi_{n} \leq \int_{-\frac{\delta}{2}}^{\frac{\delta}{2}}\|\dot{f}(.+t)\| p, \psi_{n} \mathrm{~d} t$

$\tau_{1}(f, \delta) p, \psi_{n} \leq \delta\|f\| p, \psi_{n}$

Lemma 13. Let $f \in L p, \psi_{n}(X)$, then:

$\tau_{\mathrm{k}}(f, \delta) p, \psi_{n} \leq C(k) \delta^{k}\left\|f^{(k)}\right\| p, \psi_{n}$

Proof. From Lemma 11 and Lemma 12

$$
\begin{aligned}
\tau_{\mathrm{k}}(f, \delta) p, \psi_{n} & \leq \delta \tau_{k-1}\left(\hat{f}, \frac{k}{k-1} \delta\right) p, \psi_{n} \\
& \leq \delta \tau_{k-2}\left(f, \frac{k-1}{k-2} \delta\right) p, \psi_{n} \\
& \leq \delta \tau_{k-3}\left(f, \frac{k-2}{k-3} \delta\right) p, \psi_{n} \\
& \leq \delta^{k-1} \tau_{1}\left(f^{(k-1)}, 2 \delta\right) p, \psi_{n}
\end{aligned}
$$

From Lemma 4, we get:

$$
\begin{aligned}
\tau_{\mathrm{k}}(f, \delta) p, \psi_{n} & \leq 2 \delta^{k}\left\|f^{(k)}\right\| p, \psi_{n} \\
& \leq \mathrm{c}(k) \delta^{k}\left\|f^{(k)}\right\| p, \psi_{n}
\end{aligned}
$$

Lemma 14. For even natural number $k$ and even $h>0$ there exists a function $f_{k, h} \in L p, \psi_{n}(X), \mathrm{B}=[0,2 \pi]$, then: 1- $\left|\left[f(x)-f_{k, \delta}(x)\right] \psi_{n}(x)\right| \leq c_{1} \omega_{\mathrm{k}}(f, x, \delta) p, \psi_{n}$

2- $\left\|f-f_{k, \delta}\right\| \leq c_{1} \omega_{\mathrm{k}}(f, \delta) p, \psi_{n}$

3- $\left\|f_{k, \delta}^{r}\right\| p, \psi_{n} \leq \mathrm{C}_{2} \frac{1}{\delta} \omega_{\mathrm{r}}(f, \delta) p, \psi_{n}$

Proof (1). We can define the function [8]

$$
\begin{array}{r}
f_{k, \delta}(x)=(-\delta)^{-k} \int_{0}^{\delta} \ldots \int_{0}^{\delta}\left\{-f\left(x+t, \ldots+t_{k}\right) \psi_{n}(t)\right. \\
+\left(k_{1}\right) f\left[x+\frac{k-1}{k}\left(t, \ldots+\mathrm{t}_{\mathrm{k}}\right)\right] \psi_{n}(t)+\ldots \ldots+ \\
(-1)^{\mathrm{k}}\left(\begin{array}{c}
k \\
k-1
\end{array}\right) f\left\{\left(x+\frac{t_{1}+\cdots t_{k}}{k}\right) \psi_{n}(t)\right\} \mathrm{dt}_{1} \ldots \mathrm{dt}_{2} \\
\left|\left(f-f_{k, \delta}\right) \psi_{n}(x)\right| \leq \frac{1}{-\delta} \int_{0}^{\delta} \ldots \int_{0}^{\delta}||_{\sum_{i=1}^{k}}^{\Delta^{k}}+f(x) \mid \\
\left.\leq \omega_{\mathrm{k}}(f, x ; \delta) p, \psi_{n} 2\right) f_{k, \delta} \\
=\frac{1}{(-\delta)^{k}} \int_{0}^{\delta} \ldots \int_{0}^{\delta}\left\{f\left(x+\sum_{i=1}^{k} t_{\mathrm{i}}\right) \psi_{\mathrm{n}}(t)+\right. \\
\left(\begin{array}{c}
k \\
1
\end{array}\right) f\left[x+\frac{k-1}{k}\left(\sum_{i=1}^{k} t_{\mathrm{i}}\right)\right] \psi_{\mathrm{n}}(t)+\ldots .+ \\
(-1)^{k}\left(\begin{array}{c}
k \\
k-1
\end{array}\right) f\left\{\left(x+\frac{1}{k}\left(\sum t_{i}\right) \psi_{\mathrm{n}}(\mathrm{t})\right\}\right. \\
\prod_{i-1}^{k} d t \\
\left\|f-f_{k, \delta}\right\| p, \psi_{n} \leq \frac{1}{\delta^{k}} \int_{0}^{\delta} \ldots \int_{0}^{\delta}\|\|_{i=1 t_{i}}^{\Delta^{k}} f \| p, \psi_{n} \prod_{i-1}^{k} d t \\
\left.\leq \mathrm{C}_{1} \omega_{k}(f, \delta) p, \psi_{n} 3\right) f_{k, \delta}^{(r)}(x) \\
=\frac{1}{(-\delta)^{k}} \int_{0}^{\delta} \ldots \int_{0}^{\delta}\left\{-\Delta_{\delta}^{r} f\left(x+\sum_{i=1}^{k-r} t_{k-r}\right) \psi_{n}(\mathrm{t})\right. \\
+\left(\begin{array}{c}
k \\
1
\end{array}\right)\left(\begin{array}{c}
k \\
k-1
\end{array}\right)^{\mathrm{r}} \Delta_{\frac{(k-1) \delta}{k}}^{r}\left[x+\frac{(k-1)}{k}\left(\sum_{i=1}^{k-r} t_{k-r}\right) \psi_{n}\right.
\end{array}
$$

$(\mathrm{t})+\ldots+(-1)^{\mathrm{k}}\left(\begin{array}{c}k \\ k-1\end{array}\right) \quad k_{r} \quad \Delta_{\frac{\delta}{k}}^{r} f\left(x+\frac{1}{k}\right.$ $\left.\left(\sum_{i=1}^{k-r} t_{k-r}\right)\right\} \prod_{i-1}^{k} d t_{\mathrm{i}}$ $\left\|f_{k, \delta}^{(r)}\right\|_{p, \psi_{n}} \leq \frac{1}{\delta^{k}} \int_{0}^{\delta} \ldots \int_{0}^{\delta}\left\{\| \Delta_{\delta}^{r} f(x+\right.$ $\left(\sum_{i=1}^{k-r} t_{k-r}\right) \psi_{n}(t) \|_{p, \psi_{n}}+\left(\begin{array}{c}k \\ 1\end{array}\right)\left(\begin{array}{c}k \\ k-1\end{array}\right)^{\mathrm{r}}$ $\| \Delta_{\frac{(k-1) \delta}{k}}^{r} f[x+$ $\left.\frac{k-1}{k}\left(\sum_{i=1}^{k-r} t_{k-r}\right)\right] \psi_{n}(t) \|_{p, \psi_{n}}+\ldots .+\left(\begin{array}{c}k \\ k-1\end{array}\right)$ $\left.k^{r}\left\|\Delta_{\frac{\delta}{k}}^{r} f\left[x+\frac{1}{k}\left(\sum_{i=1}^{k-r} t_{k-r}\right)\right] \psi_{n}(t)\right\|\right\}$ $\prod_{i-1}^{k} d t_{\mathrm{i}}$ $\left\|f_{k, \delta}^{(r)}\right\|_{p, \psi_{n}} \leq \frac{1}{\delta^{k}}\left\{\omega(f \delta)_{\mathrm{p},} \psi_{n}+\left(\begin{array}{c}k \\ 1\end{array}\right)\left(\begin{array}{c}k \\ k-1\end{array}\right)^{\mathrm{r}} \omega_{\mathrm{r}}(f\right.$, $\left.\left.\frac{(k-1) \delta}{k}\right) p, \psi_{n}+\ldots+\left(\begin{array}{c}k \\ k-1\end{array}\right) k^{r} \omega_{r}\left(f, \frac{\delta}{k}\right)_{p, \psi_{n}}\right\}$ $\leq \mathrm{C}_{2} \frac{1}{\delta^{r}} \omega_{r}(f, \delta)_{p, \psi_{n}}$

\section{Main Result}

Theorem 1. Let $f \in L p, \psi_{n}(X), X=[0,2 \pi]$, then: $\tilde{E}_{n}(f) \mathrm{p}, \psi_{n} \leq C \tau\left(f, n^{-1}\right) \mathrm{p}, \psi_{n}, 1 \leq \mathrm{i}<\infty$, where $C$ is a constant.

Proof. Let $x_{\mathrm{i}}=i \pi / n, \mathrm{i}=0,1, \ldots, 2 \mathrm{n}$ $y_{\mathrm{i}}=\left(x_{\mathrm{i}-1}+x_{\mathrm{i}}\right) / 2, \mathrm{i}=1,2, \ldots, 2 n$

Let us define the linear continuous $2 \pi$-periodic function $s_{n}$. and $y_{n}$ for $x \in\left[x_{i-1}, y_{i}\right]$ and for $x \in\left[y_{\mathrm{i}}, x_{\mathrm{i}}\right], i=1,2, \ldots$, $2 n, y_{\mathrm{i}}=\left(x_{\mathrm{i}-1}+x_{i}\right) / 2$

$s_{n}(x)=\sup \left\{f(\mathrm{t}) \psi_{n}(\mathrm{t}): \mathrm{t} \in\left[x_{i-1}, x_{i}\right]\right\}, x=y_{i, i} i=1,2, \ldots, 2 n$ such that:

$$
s_{n}(0)=s_{n}(2 \pi)
$$

$y_{n}(x)=\inf \left\{f(\mathrm{t}) \psi_{\mathrm{n}}(t): t \in\left[x_{i-1}, x_{i}\right]\right\}$, for $x=y_{i, i}=1, \ldots, 2 n$ such that $y_{n}(0)=y_{n}(2 \pi)$.

Obviously:

$$
y_{n}(x) \leq f(x) \leq s_{n}(x), x \in[0,2 \pi]
$$

The derivatives $S_{n}$ and $y_{n}$ of the function $y_{n}(x)$ and $s_{n}(x)$ exist in $[0,2 \pi]$ except at the points $x_{i}, i=1, \ldots 2 n, y_{i}, i=$ $1, \ldots, 2 n$. Using the definitions of the function $s_{n}$ and $y_{n}$ we obtain set $\delta=4 \pi \mathrm{n}^{-1}$

Let $x \in\left(y_{\mathrm{i}}, x_{\mathrm{i}}\right)$ since $s_{n}$ is linear in $\left(y_{\mathrm{i}}, x_{\mathrm{i}}\right)$, we have:

$$
\left|s_{n}^{\prime}(x)\right| \leq \frac{2 n}{\pi}\left|s_{n}\left(y_{i-1}\right)-s_{n}\left(y_{i}\right)\right| \leq \frac{2 n}{\pi} \omega(f, x, \delta) p, \psi_{n}
$$

Also $\left|y_{n}(x)\right| \leq C_{n}=\omega_{1}(f, x, \delta) p, \psi_{n}$ again from the definition of the $S_{n}$ and $y_{n}$, we obtain:

$0 \leq s_{n}(x)-y_{n}(x) \leq \omega(f, x, \delta) p, \psi_{n}$

$\left\|s_{n}\right\| p, \psi_{n} \leq\left\|C_{n} \omega(f, x, \delta)\right\| p, \psi_{n}$

$$
\leq C_{n} \tau_{1}(f, \delta) p, \psi_{n}
$$

$\left\|y_{n}^{\prime}\right\| p, \psi_{n} \leq\left\|C_{n} \omega_{1}(f, x, \delta)\right\| p, \psi_{n}$

$$
\leq C_{n} \tau(f, \delta) p, \psi_{n}
$$

Also:

$s_{n}(x)-y_{n}(x) \leq \omega(f, x, \delta) p, \psi_{n}$

$\left\|S_{n}(x)-y_{n}(x)\right\| p, \psi_{n} \leq\|\omega(f, x, \delta)\| p, \psi_{n}$

$$
\leq \tau_{1}(f, \delta) p, \psi_{n}
$$

Applying Lemma 8 in (1), (2), we get:

$$
\begin{aligned}
\widetilde{E}_{n}\left(s_{n}\right) p, \psi_{n} & \leq \frac{c_{1}}{n} C_{\mathrm{n}} \tau_{1}(f, \delta) p, \psi_{n} \\
& \leq C_{2} \tau_{1}(f, \delta) p, \psi_{n}
\end{aligned}
$$




\section{Al-Nahrain Journal of Science}

ANJS, Vol.24 (4), December, 2021, pp. 40-45

Also:

$$
\tilde{E}_{n}\left(y_{n}\right) p, \psi_{n} \leq \frac{c_{1}}{n} C_{n} \tau_{1}(f, \delta) p, \psi_{n}
$$$$
\leq C_{2} \tau_{1}(f, \delta) p, \psi_{n}
$$

Sine $\tilde{E}_{n}(f) p, \psi_{n}=\inf \left\|s_{n}-y_{n}\right\| p, \psi_{n}$

$\tilde{E}_{n}(f) p, \psi_{n} \leq\left\|s_{n}-y_{n}\right\| p, \psi_{n}$

$$
\begin{aligned}
\leq & \widetilde{E}_{n}\left(s_{n}\right) p, \psi_{n}+\left\|s_{n}-y_{n}\right\| p, \psi_{n}+ \\
& \widetilde{E}_{n}\left(y_{n}\right) p, \psi_{n}
\end{aligned}
$$

From (3), (4) and (5), we get:

$$
\begin{aligned}
\tilde{E}_{n}(f) p, \psi_{n} \leq & C_{n} \tau(f, \delta) p, \psi_{n}+\tau_{1}(f, \delta) p, \psi_{n}+ \\
& C_{n} \tau_{1}(f, \delta) p, \psi_{n} \\
\leq & \left(2 C_{1}+1\right) \tau_{1}(f, \delta) p, \psi_{n} \\
\leq & C \tau_{1}(f, \delta) p, \psi_{n}
\end{aligned}
$$

where $2 C_{2}+1=C$ is a constant.

\section{Direct theorem}

Theorem 2. Let $f \in L p, \psi_{n}(X)$, for every natural number $k$ there is a constant $C(k)$, then:

$$
\tilde{E}_{n}(f) p, \psi_{n} \leq C(k) \tau_{k}\left(f, \frac{1}{n}\right) p, \psi_{n}
$$

Proof. Applying Lemma 3 and Lemma 10 to the functions $f, f_{k, \delta}$ and

$$
\begin{aligned}
& \varphi(x)=\omega_{k}\left(f, 2 n^{-1}\right) p, \psi_{n} \\
& \widetilde{E}_{n}(f) p, \psi_{n}=C_{p}\left(\widetilde{E}_{n}\left(f_{k, \delta}\right) p, \psi_{n}+2 \widetilde{E}_{n}(\varphi) p, \psi_{n}+2\right. \\
& \| \omega_{k}\left(f, x, \delta \| p, \psi_{n}\right. \\
& \leq C_{1}\left(\widetilde{E}_{n}\left(f_{k, \delta}\right) p, \psi_{n}+2 C p \tau_{k}(f, \delta) p, \psi_{n}\right. \\
& \quad 2 C_{p} \tau_{k}(f, \delta) p, \psi_{n} \\
& \tilde{E}_{n}\left(f_{k, \delta}\right) p, \psi_{n} \leq C(k)\left\|f_{k, \delta}^{(k)}\right\| p, \psi_{n} \\
& \leq C_{1}(k) \omega_{k}(f, \delta) p, \psi_{n} \\
& \leq C_{2}(k) \tau_{k}(f, \delta) p, \psi_{n}
\end{aligned}
$$

From (6) and (7), then:

$$
\begin{aligned}
\tilde{E}_{n}(f) p, \psi_{n} \leq & \mathrm{C}_{2}(k) \tau_{k}(f, \delta) p, \psi_{n}+2 \mathrm{C}_{\mathrm{p}} \tau_{k}(f, \delta) \\
& p, \psi_{n}+2 \mathrm{C}_{\mathrm{p}} \tau_{k}(f, \delta) p, \psi_{n} \\
\leq & 4 \mathrm{C}_{\mathrm{p}} \mathrm{C}_{2}(k) \tau_{k}(f, \delta) p, \psi_{n} \\
\leq & \mathrm{C}(\mathrm{p}, k) \tau_{k}(f, \delta) p, \psi_{n}
\end{aligned}
$$

Theorem 3. Let $f \in L p, \psi_{n}(X)$, there exists $C(k)$

$$
\tau_{k}\left(f, n^{-1}\right) p, \psi_{n} \leq \frac{C(k)}{n^{k}} \sum_{s=0}^{n}(s+1)^{k-1} \tilde{E}_{n}(f) p, \psi_{n}
$$

Proof. Let $\alpha_{n}, \gamma_{n}, \in T_{n}$ are trigonometric polynomials

$\tilde{E}_{n}(f) p, \psi_{n}=\inf \left\|\alpha_{n}-\gamma_{n}\right\| p, \psi_{n}, \gamma_{n}(x) \leq f(x) \leq \alpha_{n}$

$$
(x), x \in[0,2 \pi]
$$

If $\Delta_{h}^{k} f(\mathrm{t}) \psi_{n}(\mathrm{t}) \geq 0$, then:

$$
\begin{aligned}
\Delta_{h}^{k} f(\mathrm{t}) \psi_{n}(\mathrm{t})= & \sum_{m=0}^{k}(-1)^{m}\left(\begin{array}{l}
k \\
m
\end{array}\right) f[\mathrm{t}+(k-m) h] \psi_{n}(t) \\
\leq & \sum_{i=0}^{k / 2}\left(\begin{array}{c}
k \\
2 i
\end{array}\right) \alpha_{n}[\mathrm{t}+(k-2 \mathrm{i}) h]- \\
& \sum_{i=0}^{k-1 / 2}\left(\begin{array}{c}
k \\
2 i+1
\end{array}\right) \gamma_{n}[\mathrm{t}+(k-2 \mathrm{i}-1) h] \\
= & \Delta_{h}^{k} \alpha_{n}(\mathrm{t})+\sum_{i=0}^{k-1 / 2}\left(\begin{array}{c}
k \\
2 i+1
\end{array}\right)\left\{\gamma_{n}[\mathrm{t}+(k-\right. \\
& \left.2 \mathrm{i}-1) h]-\alpha_{n}[t+(k-2 \mathrm{i}-1) h]\right\} \\
= & \Delta_{h}^{k} \alpha_{n}(\mathrm{t})+\sum_{i=0}^{k-1 / 2}\left(\begin{array}{c}
k \\
2 i+1
\end{array}\right)\left\{\alpha_{n}[\mathrm{t}+\right. \\
& (k-2 \mathrm{i}-1) h-\alpha_{n}[\mathrm{t}+(k-2 \mathrm{i}- \\
& \left.1) h]-\left[\alpha_{n}(x)-\gamma_{n}(x)\right]\right\}+ \\
& \sum_{i=0}^{k-1 / 2}\left(\begin{array}{c}
k \\
2 i+1
\end{array}\right)\left[\alpha_{n}(x)-\gamma_{n}(x)\right] \\
\leq & \Delta_{h}^{k} \alpha_{n}(\mathrm{t})+2^{k}\left\{\omega_{1}\left(\alpha_{n}-\gamma_{n}, x ; k \delta\right)\right. \\
& \left.p, \psi_{n}+\left[\alpha_{n}(x)-\gamma_{n}(x)\right]\right\}
\end{aligned}
$$

$$
\begin{array}{r}
\left|\Delta_{h}^{k} f(\mathrm{t}) \gamma_{n}(\mathrm{t})\right| \leq\left|\Delta_{h}^{k} \alpha_{n}(\mathrm{t})\right|+2^{k}\left[\omega_{1}\left(\alpha_{n}-\gamma_{n}, x ; k \delta\right)\right. \\
\left.p, \psi_{n}+\left|\alpha_{n}(x)-\gamma_{n}(x)\right|\right]
\end{array}
$$

Now if $\Delta_{h}^{k} f(\mathrm{t}) \psi_{n}(\mathrm{t}) \leq 0$, then in the same way, we obtain:

$$
\begin{aligned}
& \left|\Delta_{h}^{k} f(\mathrm{t}) \psi_{n}(\mathrm{t})\right| \leq\left|\Delta_{h}^{k} \gamma_{n}(\mathrm{t})\right|+2^{k}\left[\omega_{1}\left(\alpha_{n}-\gamma_{n}, x ; k \delta\right)\right. \\
& p, \psi_{n}+\left[\alpha_{n}(x)-\gamma_{n}(x)\right]
\end{aligned}
$$

From equations (8) and (9), we get

$$
\begin{gathered}
\omega_{k}(f, x, \delta)_{p, \psi_{n}} \leq \omega_{k}\left(\alpha_{n}, x \delta\right) p, \psi_{n}+\omega_{k}\left(\gamma_{n}, x, \delta\right) \\
p, \psi_{n}+2^{k}\left[\omega_{1}\left(\alpha_{n}-\gamma_{n}, x, \delta\right) \mathrm{p}, \psi_{n}+\right. \\
\left.\left|\alpha_{n}(\mathrm{x})-\gamma_{n}(x)\right|\right] \\
\tau_{k}(f, \delta)_{p, \psi_{n}} \leq \\
\tau_{k}\left(\alpha_{n}, \delta\right) p, \psi_{n}+\tau_{k}\left(\gamma_{n}, \delta\right) p, \psi_{n}+ \\
2^{k}\left[\tau_{1}\left(\alpha_{n}-\gamma_{n}, k \delta\right) p, \psi_{n}+\widetilde{E}_{n}(f)\right. \\
\left.p, \psi_{n}\right]
\end{gathered}
$$

From Lemma 4, we get:

$$
\tau_{1}\left(\alpha_{n}-\gamma_{n}, k \delta\right) p, \psi_{n} \leq k \delta\left\|\alpha_{n}^{\prime}-\gamma_{n}^{\prime}\right\| p, \psi_{n}
$$

Using the Bernstein inequality:

$$
\left\|T^{\prime}\right\|_{\mathrm{p}, \psi_{n}} \leq \mathrm{n}\|T\|_{\mathrm{p}, \psi_{n}}
$$

Thus:

$$
\begin{aligned}
\tau_{1}\left(\alpha_{n}-\gamma_{n}, k \delta\right) \mathrm{p}, \psi_{n} & \leq n k \delta\left\|\alpha_{n}-\gamma_{n}\right\|_{\mathrm{p}, \psi_{n}} \\
& =k \delta n \widetilde{E}_{n}(f) \mathrm{p}, \psi_{n}
\end{aligned}
$$

Equation (10) implies

$$
\begin{gathered}
\tau_{k}(f, \delta) p, \psi_{n} \leq \tau_{k}\left(\alpha_{n}, \delta\right) p, \psi_{n}+\tau_{k}\left(\gamma_{n}, \delta\right) p, \psi_{n}+ \\
2^{k}(k \delta n+1) E_{n}^{\sim}(f) p, \psi_{n}
\end{gathered}
$$

Let us set $n=2^{s_{0}}$, then:

$$
\begin{aligned}
\tau_{k}(f, \delta) p, \psi_{n} \leq & \sum_{i=1}^{S_{0}}\left[\tau_{k}\left(\alpha_{2^{i}}-\alpha_{2^{i}}, \delta\right) p, \psi_{n}+\right. \\
& \tau_{k}\left(\gamma_{2^{i}}-\gamma_{2^{i-1}}, \delta\right] p, \psi_{n}+\tau_{k}\left(\alpha_{1}-\alpha_{0},\right. \\
& \delta) p, \psi_{n} \tau_{k}\left(\gamma_{1}-\gamma_{0}, \delta\right) p, \psi_{n}{ }^{+} \\
& 2^{k}(k \delta n+1) E_{n}^{\sim}(f) p, \psi_{n}
\end{aligned}
$$

Now

$$
\begin{aligned}
& \tau_{k}\left(\alpha_{2^{i}}-\alpha_{2^{i-1}}, \delta\right) p, \psi_{n} \leq k \delta^{k}\left\|\left(\alpha_{2^{i}}-\alpha_{2^{i-1}}\right)^{k}\right\|_{p, \psi_{n}} \\
& \leq k \delta^{k} 2^{i k}\left\|\alpha_{2^{i}}-\alpha_{2^{i-1}}\right\|_{p, \psi_{n}} \\
& \leq k \delta^{k} 2^{i k}\left[\left\|\alpha_{2^{i}}-f\right\| p, \psi_{n}+\left\|\alpha_{2^{i-1}}-f\right\| p, \psi_{n}\right] \\
& \leq k \delta^{k} 2^{i k}\left[\left\|\alpha_{2^{i}}-\gamma_{2^{i}}\right\| p, \psi_{n}+\| \alpha_{2^{i-1}}-\right. \\
&\left.\quad \gamma_{2^{i-1}} \| p, \psi_{n}\right] \\
& \leq 2 k \delta^{k} 2^{i k} \widetilde{E}_{2^{i-1}}(f) p, \psi_{n} \\
& \tau_{k}\left(\gamma_{2^{i}}-\gamma_{2^{i-1}}\right) p, \psi_{n} \leq k \delta^{k}\left\|\left(\gamma_{2^{i}}-\gamma_{2^{i-1}}\right)^{k}\right\|_{p, \psi_{n}} \\
& \leq k \delta^{k} 2^{i k}\left\|\gamma_{2^{i}}-\gamma_{2^{i-1}}\right\|_{p, \psi_{n}} \\
& \leq k \delta^{k} 2^{i k}\left[\left\|\gamma_{2^{i}}-f\right\| p, \psi_{n}+\left\|\gamma_{2^{i-1}}-f\right\| p, \psi_{n}\right] \\
& \leq k \delta^{k} 2^{i k}\left[\left\|\alpha_{2^{i}}-\gamma_{2^{i}}\right\| p, \psi_{n}+\| \alpha_{2^{i-1}}-\right. \\
&\left.\gamma_{2^{i-1}} \| p, \psi_{n}\right] \\
& \leq 2 k \delta^{k} 2^{i k} \widetilde{E}_{2^{i-1}}(f) p, \psi_{n}
\end{aligned}
$$

From inequalities (11), (12) and (13), we get:

$$
\begin{aligned}
\tau_{k}(f, \delta) p, \psi_{n} \leq & 4 k \delta^{k} \sum_{i=1}^{s_{0}} 2^{i k} \widetilde{E}_{2^{i-1}}(f) p, \psi_{n}+ \\
& 2 k \delta^{k} E_{0}^{\sim}(f) p, \psi_{n}+2^{k}\left(k \delta_{n+1}\right) \\
& \widetilde{E}_{n}(f) p, \psi_{n}
\end{aligned}
$$

Let $\delta=n^{-1}$, then:

$$
\begin{gathered}
\tau_{k}(f, \delta) p, \psi_{n} \leq 4^{k+1} k n^{-k} \sum_{s=0}^{n}(s+1)^{k-1} \tilde{E}_{s}(f) \\
p, \psi_{n}+2^{k}(k+1) \tilde{E}_{n}(f) p, \psi_{n} \\
\leq 2^{3 k+1} n^{-k} \sum_{s=0}^{n}(s+1)^{k-1} \widetilde{E}_{s}(f) p, \psi_{n} \\
\tau_{k}(f, \delta) p, \psi_{n} \frac{c(k)}{n^{k}} \sum_{s=0}^{n}(s+1)^{k-1} \widetilde{E}_{s}(f) p, \psi_{n}
\end{gathered}
$$




\section{Al-Nahrain Journal of Science}

ANJS, Vol.24 (4), December, 2021, pp. 40-45

\section{References}

[1] Babenko A. G.; "One-sided approximation in L of the characteristic function of an interval by trigonometric polynomials". Trudy institnte mathematika mekhanki, Uro RAN m, 18(1): 88 -95, 2012.

[2] Soltani F.; "Best approximation of the dunkl multiplier operators $\mathrm{T}_{\mathrm{k}, 1, \mathrm{~m}}$. International Journal of analysis and applications, 7(2), 2015.

[3] Jassim S. K. and Abeer M. S.; "Multiplier approximation of unbounded functions by Bernstien Durrmeyer operators", M.Sc. Thesis, University of AlMustansiriya, College of Science, Department of Mathematics, 2017.

[4] Sahib Al-Saidy and Ali H. Z.; "Best Multiplier approximation of Periodical unbounded functions using trigonometric operators". M. Sc. Thesis, University of Al-Mustansiriya, College of Science, Department of Mathematics, 2020.

[5] Pasko A. M.; "The point wise estimation of the onesided approximation of the class $W_{\infty}^{r}, 0<r<1$." Res. Math. 28(1): 22-28, 2022.

[6] Haoguo and Rongluli; "Multiplier convergent series and uniform convergence of mapping series " proc. Indian Acad. Sci. (Math. Sci.), 123(1): February, 67-73, 2013.

[7] Vladislav K.; Dzyadyk I. and Shevchuk A.; "Theory of uniform approximation of functions by polynomials". 2008.

[8] Sendov B. C. and Popov V. A.; "The averaged moduli of smoothness: applications in numerical method and approximation". Chi Chester, 1988 